\title{
Association between red blood cell storage duration and clinical outcome in patients undergoing off-pump coronary artery bypass surgery: a retrospective study
}

\author{
Jeong Jin Min ${ }^{1,2}$, Jun-Yeol Bae ${ }^{1}$, Tae Kyong Kim, Deok Man Hong ${ }^{1 *}$, Ho Young Hwang ${ }^{3}$, Ki-Bong Kim³
} Kyou-Sup $\mathrm{Han}^{4}$ and Yunseok Jeon ${ }^{1 *}$

\begin{abstract}
Background: Prolonged storage of red blood cells (RBCs) leads to fundamental changes in both the RBCs and the storage media. We retrospectively evaluated the relationship between the RBC age and in-hospital and long-term postoperative outcomes in patients undergoing off-pump coronary artery bypass.

Methods: The electronic medical records of 1,072 OPCAB patients were reviewed and information on the transfused RBCs and clinical data were collected. The effects of RBCs age (mean age, oldest age of transfused RBCs, any RBCs older than 14 days) on various in-hospital postoperative complications and long-term major adverse cardiovascular and cerebral events over a mean follow-up of 31 months were investigated. Correlations between RBCs age and duration of intubation, intensive care unit, or hospital stay, and base excess at the first postoperative morning were also analyzed.

Results: After adjusting for confounders, there was no relationship between the RBCs age and in-hospital and long-term clinical outcomes except for postoperative wound complications. A significant linear trend was observed between the oldest age quartiles of transfused RBCs and the postoperative wound complications (quartile 1 vs. 2, 3 and 4: OR, 8.92, 12.01 and 13.79 , respectively; $P$ for trend $=0.009$ ). The oldest transfused $\mathrm{RBC}$ s showed significant relationships with a first postoperative day negative base excess $(P=0.021)$, postoperative wound complications $(P=0.001)$, and length of hospital stay $(P=0.008)$.

Conclusions: In patients undergoing off-pump coronary artery bypass, the oldest age of transfused RBCs were associated with a postoperative negative base excess, increased wound complications, and a longer hospital stay, but not with the other in-hospital or long-term outcomes.
\end{abstract}

Keywords: RBC storage age, Old stored RBC, Postoperative outcome, Wound complication, Cardiac surgery

\section{Background}

Prolonged storage of red blood cells (RBCs) alters them and their storage media, causing changes referred to as 'storage lesions'. Over time, intracellular adenosine triphosphate within the stored RBCs decreases, rendering the RBC membrane fragile and less deformable [1]. The breakdown of fragile RBCs releases free hemoglobin and microparticles which reduce nitric oxide bioavailability,

\footnotetext{
*Correspondence: mellyn7@gmail.com; jeonyunseok@gmail.com

'Department of Anesthesiology and Pain Medicine, Seoul National University Hospital, Seoul, Korea

Full list of author information is available at the end of the article
}

leading to vasoconstriction, thrombosis, and inflammation [2,3]. Moreover, depleted 2,3 DPG decreases oxygen delivery to organs [4]. Numerous studies on various patient populations have investigated the clinical impact of RBC storage lesions. However, their impact is still debatable.

Several studies on cardiac surgery patients have investigated the association between clinical outcomes and the storage time of RBCs [5-9]. However, previous studies used heterogeneous populations that included patients who received open heart surgery for valvular heart disease or an on-pump coronary artery bypass. Valvular heart diseases have various cardiac pathophysiologies according to 
the disease type and severity. Moreover, cardiopulmonary bypass with hypothermia is associated with inflammatory, metabolic, and hematologic responses and various organ injuries, making elucidation of the effects of old stored blood in such patient populations more complex [10]. Although a few studies have investigated the clinical effects of transfusions of old stored blood in patients undergoing coronary artery bypass surgery with regard to vasoconstrictive, thrombotic, and inflammatory effects [3,5,7-9,11], to our knowledge, the effects of stored RBCs have not been investigated exclusively in patients undergoing off-pump coronary artery bypass (OPCAB) surgery.

Although the transfusion rate of RBCs in off-pump CABG surgery was lower than that in on-pump surgery, more than half of the OPCAB patients still needed RBCs transfusion [12]. In patients with coronary arterial disease, RBCs transfusion is essential for adequate oxygen delivery. Meanwhile, old blood transfusion is a concern in such patients because of the possible harmful vascular effects $[2,3]$.

We hypothesized that prolonged storage of RBCs may be associated with adverse in-hospital and long-term postoperative outcomes in patients undergoing OPCAB. To evaluate this hypothesis, we retrospectively studied the relationship between the RBC storage duration and in-hospital clinical outcomes and long-term postoperative major adverse cardiovascular and cerebral events (MACCEs) in patients undergoing OPCAB.

\section{Methods}

The study protocol was approved by the institutional review board of our hospital (IRB No. 1302-052-465, Seoul National University Hospital). As this was a retrospective study using electronic medical records, individual informed consent was waived. We screened the computerized medical records of 1,113 patients who underwent $\mathrm{OPCAB}$ between December 2005 and May 2012 and identified patients who received $\mathrm{RBC}$ transfusions during their hospital stay. A total of 41 patients who had not received RBC transfusions were excluded. Therefore, the final study population included 1,072 patients.

\section{Data collection}

The electronic medical records of enrolled patients were reviewed and pre-, intra-, and postoperative data were collected by researchers who were not aware of the RBC transfusion information. The clinical follow-up concluded in September 2012, with a mean follow-up duration of 31 (inter-quartile range [IQR], 11-51) months.

To determine the quantity and age of RBCs, information about all RBC units transfused to enrolled patients during their hospital stay was obtained from the computerized database of our institutional blood bank with the aid of the hospital's Medical Information Department. The storage time (in days) of RBCs was analyzed in three ways: (1) the mean age of transfused RBCs units, (2) the oldest age of transfused RBCs units, and (3) any transfusion of RBCs units older than 14 days as a categorical variable.

All RBCs units were provided by the Korean Red Cross Blood Services. The RBCs were stored in citrate phosphate dextrose adenine (CPDA)-1 and the storage temperature was $2-6^{\circ} \mathrm{C}$. RBCs units in our institution's blood bank are discarded after 35 days of storage. The perioperative coagulation management strategy was as follows: all patients took aspirin until the day of the surgery and resumed it as soon as possible after the surgery, usually one day postoperatively. During the surgery, the patients were given an initial dose of heparin $(1.5 \mathrm{mg} / \mathrm{kg})$ and periodic supplemental doses to maintain an ACT $>300 \mathrm{sec}$. Heparin was neutralized at the end of the surgery to only one-third of the required protamine dose. The perioperative target hemoglobin level was $10 \mathrm{~g} / \mathrm{dl}$.

\section{Study end points and definition}

The primary endpoint was the in-hospital and long-term MACCEs, defined as a composite of death from cardiac causes, myocardial infarction (MI), coronary revascularization, and stroke. The long-term follow-up was initiated after hospital discharge and concluded in September 2012. The mean follow-up period was 31 months, with the range 0 to 80 months (median 29, inter-quartile range [IQR], 11-51 months). Other study endpoints were in-hospital postoperative adverse outcomes including all-cause mortality, new renal failure, respiratory complications, postoperative wound complications, a new arrhythmia requiring treatment, bleeding-related reoperations, and the length of ICU and hospital stay. Definitions of each in-hospital postoperative outcome are as follows; Death was considered to be of cardiac origin if attributed to myocardial infarction, cardiac arrhythmia, or heart failure caused primarily by a cardiac problem. MI or stroke diagnosis and coronary revascularization were confirmed by reviewing hospital records. Respiratory complications included prolonged ventilator support $(>48 \mathrm{~h})$ or postoperative pneumonia. The diagnosis of pneumonia was based on a combination of physical signs and a chest X-ray and often confirmed by microbiological tests. Postoperative new renal failure was defined as an increase of $>50 \%$ in serum creatinine from the preoperative value or the requirement for new renal replacement therapy regardless of serum creatinine level. Postoperative wound complication was defined as any sternal wound complication after surgery such as superficial and deep sternal wound including mediastinitis. Arrhythmias other than atrial fibrillation were defined as a postoperative new arrhythmia requiring treatment, including frequent multifocal premature ventricular contractions, ventricular bigemini or quadrigemini, junctional rhythm, paroxysmal 
supraventricular tachycardia, ventricular tachycardia, ventricular fibrillation and asystole. Bleeding-related reoperation was confirmed by reviewing hospital records.

\section{Statistical analysis}

Continuous variables are presented as the mean (SD) and categorical variables as numbers and percentages. Linearity assumptions in the continuous variables were examined using restricted cubic splines. After checking for violation of the proportional hazard assumption, Cox proportional hazards regression models were used to identify the univariate and multivariable covariates associated with long-term MACCEs. Hazard ratios (HRs) and $95 \%$ confidence intervals (CIs) were calculated for each factor using Cox proportional hazards analysis. To assess the independent impact of each risk factor on various postoperative outcomes, univariate and multivariable logistic regression models were constructed.

Variables that included as risk factors for adverse postoperative outcomes were as follows: total number of transfused RBCs, patient's age, sex, body mass index, presence of diabetes mellitus, hypertension, dyslipidemia, previous history of myocardial infarction, previous history of stroke, presence of renal failure, left ventricular dysfuction (LV ejection fraction less than 35\%), chronic obstructive pulmonary disease, cardiac reoperation, perioperative IABP insertion, emergency operation and the duration of surgery. In the Cox regression model for MACCEs, perioperative use of statin, anesthetic agent, lowest values of intraoperative hemodynamic variables and lowest hematocrit were also included as covariates.

All adjusted models were constructed using the forward variable selection method and a forward selection criterion for model fit of $P=0.1$ was used. To determine the effect of RBC age on in-hospital and long-term clinical outcomes, we constructed each adjusted model including each $\mathrm{RBC}$ age as a covariate after forward variable selection although they showed insignificant results in the univariate analysis.

Pearson's correlation or Spearman's rank correlation coefficients were used as appropriate to analyze the relationships between RBC transfusion amount and some continuous variables such as postoperative base excess, total bilirubin, length of ICU and hospital stay. Partial correlation analyses were used to remove the effects of the number of transfused RBCs in the relationships between the three ages of RBCs and those continuous variables. A $P$ value of $<0.05$ was considered to indicate statistical significance. Analyses were performed using SPSS 19.0 (SPSS, Chicago, IL).

\section{Results}

Baseline and operative characteristics of the 1,072 patients studied are shown in Table 1. Although there was a male
Table 1 Baseline and operative characteristics

\begin{tabular}{lc}
\hline Patient variables & \\
\hline Number of patients & 1072 \\
Age, yr & $65(9)$ \\
Male & $766(71.5 \%)$ \\
Body mass index $\left(\mathrm{kg} \mathrm{m}^{-2}\right)$ & $25(3)$ \\
Diabetes mellitus & $528(49.3 \%)$ \\
Hypertension & $771(71.9 \%)$ \\
Dyslipidemia & $246(22.9 \%)$ \\
Previous myocardial infarction & $86(8.0 \%)$ \\
Previous stroke & $155(14.5 \%)$ \\
Acute renal failure & $17(1.6 \%)$ \\
Chronic renal failure & $156(14.6 \%)$ \\
LV dysfunction (EF <35\%) & $151(14.1 \%)$ \\
COPD & $12(1.1 \%)$ \\
Previous cardiac surgery & $35(3.3 \%)$ \\
Preoperative IABP insertion & $218(20.3 \%)$ \\
Operative variables & \\
Emergent operation & $181(16.9 \%)$ \\
Number of coronary grafts & $3(1)$ \\
Duration of surgery, min & $370(77)$ \\
\hline Da are presented as mean (SD) or numbr
\end{tabular}

Data are presented as mean (SD) or number (\%).

LV, left ventricle; EF, ejection fraction; COPD, chronic obstructive pulmonary disease; IABP, intra-aortic balloon pump.

dominance (71.6\%), there was no sex-related difference in the transfused RBCs amounts. A total of 7,480 allogenic, non-leukoreduced RBC units were transfused in the 1,072 patients. The mean storage time of the transfused RBCs was 11.7 (5.2) days (range, 0-35 days); the distribution of the storage time of all transfused RBCs units is shown in Figure 1. The average number of transfused RBCs per patient was 7 (8) units and the distribution of the number of transfused RBCs and the average values of RBCs age according to the number of transfused RBCs units are shown in Figure 2. In all, 473 patients (44\%) received at least one RBC unit that was older than 14 days.

\section{Long-term MACCEs}

MACCEs occurred in $16.9 \%$ (181/1072) of patients during the follow-up period, including 15 deaths from cardiac causes, 48 myocardial infarctions, 88 coronary revascularizations, and 44 strokes. Some patients had more than one complication. The detailed description of the components of MACCEs was presented in the Additional file 1. In univariate analysis, MACCEs were significantly associated with the amount of RBCs transfused but not with the storage time of RBCs (Table 2). After adjusting for the variables with $P<0.1$ in the univariate analyses, total number of transfused RBCs (HR, 1.03; 95\% CI, 1.021.04; $\mathrm{P}<0.001)$, and the use of postoperative statin $(\mathrm{HR}$, 


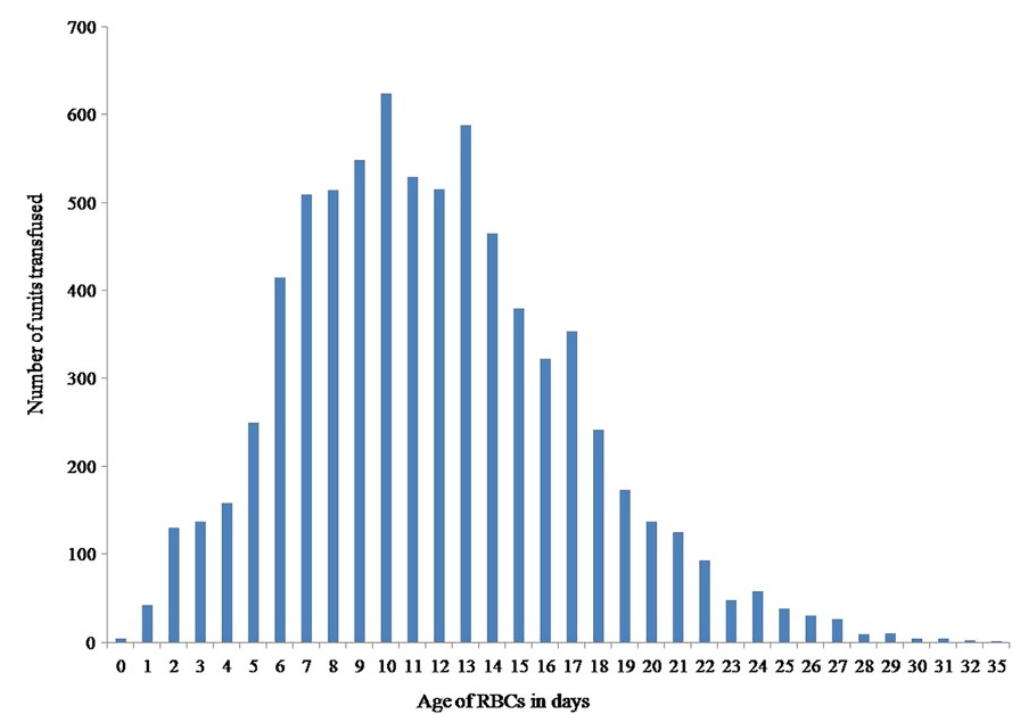

Figure 1 Storage age of all transfused RBCs. The figure shows the distribution of RBCs age and the number of transfusions for each RBCs age. $\mathrm{RBC}$, red blood cell.

0.56; 95\% CI, 0.37-0.86; $\mathrm{P}=0.007$ ) were the factors significantly associated with MACCEs. We constructed separate adjusted models including those two significant factors and the age of RBCs as covariates, which revealed no significant association between RBCs age and longterm MACCEs (Table 2).

\section{In-hospital complications}

The incidence of each postoperative clinical outcome is presented in Table 3. The oldest transfused RBCs were strongly associated with all of the in-hospital postoperative adverse outcomes except for MACCEs in the univariate analyses but the predictive effects disappeared in multivariable analyses adjusting for confounders, except for postoperative wound complications (Table 3, see also Additional file 2). For postoperative wound complications, oldest age of transfused RBCs (OR, 1.09; 95\% CI, 1.041.14; $\mathrm{P}=0.001)$, total number of transfused $\mathrm{RBCs}(\mathrm{OR}$, 1.03; 95\% CI, 1.01-1.06; $\mathrm{P}=0.001$ ), presence of diabetes mellitus (OR, 1.96; 95\% CI, 1.05-3.67; $\mathrm{P}=0.035)$, and body mass index (OR, 1.14; 95\% CI, 1.03-1.25; P =0.008) were significantly associated on multivariable analysis (Additional file 2). The number of transfused RBCs was significantly correlated with all of the analyzed clinical outcomes even after adjusting for confounding parameters (Table 3, Additional file 2). Mean age of transfused RBCs was not significantly related with any of the postoperative clinical outcomes. A significant association between 'any RBCs unit $>14$ days' and some postoperative outcomes (postoperative wound complications and bleeding-related reoperation) disappeared after adjusting for confounders (Table 3).

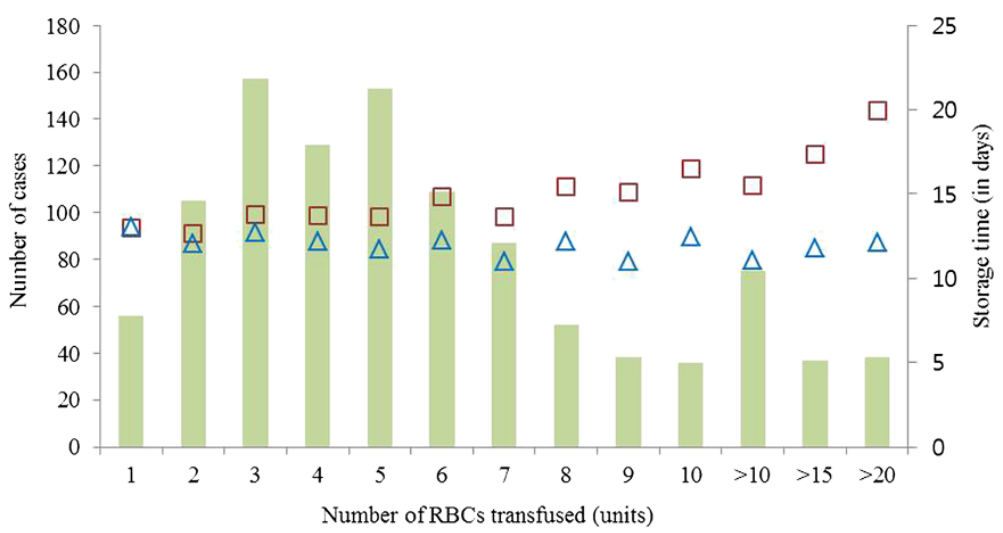

Figure 2 Transfusion amounts per patient and its relationship with the RBCs age. Bars represent the number of cases for each transfusion amounts. In each number of transfusions, squares $(\square)$ represent the average value of 'oldest age of transfused RBCs' and triangles $(\triangle)$ represent the average value of 'mean age of transfused RBCs'. RBC, red blood cell. 
Table 2 Predictors for MACCEs

\begin{tabular}{|c|c|c|c|c|c|c|c|c|c|c|c|c|}
\hline & \multicolumn{3}{|c|}{ Univariable analysis } & \multicolumn{3}{|c|}{ Adjusted model $1^{*}$} & \multicolumn{3}{|c|}{ Adjusted model $2^{\dagger}$} & \multicolumn{3}{|c|}{ Adjusted model $3^{\ddagger}$} \\
\hline & HR & $95 \% \mathrm{Cl}$ & $P$ value & $\mathrm{HR}$ & $95 \% \mathrm{Cl}$ & $P$ value & HR & $95 \% \mathrm{Cl}$ & $P$ value & $\mathrm{HR}$ & $95 \% \mathrm{Cl}$ & $P$ value \\
\hline Total number of RBCs transfusion & 1.04 & $1.03-1.05$ & $<0.001$ & 1.03 & $1.02-1.04$ & $<0.001$ & 1.03 & $1.02-1.04$ & $<0.001$ & 1.03 & $1.02-1.04$ & $<0.001$ \\
\hline Mean RBCs age & 0.97 & $0.94-1.00$ & 0.053 & 0.98 & $0.95-1.01$ & 0.248 & & & & & & \\
\hline Maximum RBCs age & 1.01 & $0.99-1.04$ & 0.368 & & & & 1.001 & $0.97-1.03$ & 0.948 & & & \\
\hline Any RBCs unit $>14$ days & 1.01 & $0.75-1.35$ & 0.957 & & & & & & & 0.89 & $0.65-1.24$ & 0.504 \\
\hline Age & 1.03 & $1.01-1.04$ & 0.005 & & & & & & & & & \\
\hline Male & 0.94 & $0.68-1.28$ & 0.679 & & & & & & & & & \\
\hline Body mass index $\left(\mathrm{kg} \mathrm{m}^{-2}\right)$ & 0.99 & $0.95-1.04$ & 0.652 & & & & & & & & & \\
\hline Smoking & 0.92 & $0.69-1.23$ & 0.563 & & & & & & & & & \\
\hline Diabetes mellitus & 0.9 & $0.67-1.21$ & 0.493 & & & & & & & & & \\
\hline Hypertension & 1.4 & $0.99-1.98$ & 0.059 & & & & & & & & & \\
\hline Dyslipidemia & 1.13 & $0.81-1.58$ & 0.47 & & & & & & & & & \\
\hline Previous myocardial infarction & 1.05 & $0.62-1.78$ & 0.852 & & & & & & & & & \\
\hline Previous stroke & 1.27 & $0.87-1.88$ & 0.221 & & & & & & & & & \\
\hline Acute renal failure & 1.97 & $0.73-5.33$ & 0.179 & & & & & & & & & \\
\hline Chronic renal failure & 1.6 & $1.1-2.34$ & 0.015 & & & & & & & & & \\
\hline LV dysfunction (EF < 35\%) & 1.46 & $0.99-2.14$ & 0.053 & & & & & & & & & \\
\hline COPD & 2.83 & $1.05-7.62$ & 0.04 & & & & & & & & & \\
\hline Previous cardiac surgery & 1.24 & $0.58-2.65$ & 0.572 & & & & & & & & & \\
\hline Perioperative IABP insertion & 1.77 & $1.30-2.40$ & $<0.001$ & & & & & & & & & \\
\hline Emergent operation & 1.59 & $1.13-2.24$ & 0.008 & & & & & & & & & \\
\hline Duration of surgery (min) & 1 & $1.00-1.00$ & 0.093 & & & & & & & & & \\
\hline preoperative statin use & 0.88 & $0.65-1.17$ & 0.35 & & & & & & & & & \\
\hline postoperative statin use & 0.49 & $0.32-0.75$ & 0.001 & 0.56 & $0.37-0.85$ & 0.007 & 0.56 & $0.37-0.86$ & 0.007 & 0.56 & $0.37-0.85$ & 0.006 \\
\hline anesthetics (sevoflurane vs. propofol) & 0.82 & $0.59-1.15$ & 0.26 & & & & & & & & & \\
\hline lowest intraoperative heart rate & 0.99 & $0.98-1.01$ & 0.828 & & & & & & & & & \\
\hline lowest intraoperative mean arterial pressure & 1 & $0.99-1.01$ & 0.87 & & & & & & & & & \\
\hline $\begin{array}{l}\text { lowest intraoperative mixed } \\
\text { venous saturation }\end{array}$ & 1.02 & $0.99-1.04$ & 0.24 & & & & & & & & & \\
\hline lowest intraoperative cardiac index & 0.8 & $0.48-1.35$ & 0.4 & & & & & & & & & \\
\hline lowest perioperative hematocrit & 0.97 & $0.92-1.01$ & 0.129 & & & & & & & & & \\
\hline
\end{tabular}

*Adjusted model 1 was constructed including the mean RBCs age as a covariate.

${ }^{\dagger}$ Adjusted model 2 was constructed including the oldest RBCs age as a covariate.

${ }^{\ddagger}$ Adjusted model 3 was constructed including the 'any RBCs unit $>14$ days' as a covariate.

MACCE, major adverse cardiovascular and cerebral event; RBC, red blood cell; LV, left ventricle; EF, ejection fraction; COPD, chronic obstructive pulmonary disease; $I A B P$, intra-aortic balloon pump.

\section{ICU and hospital length of stay}

Total number of transfused RBCs was significantly related with the duration of ICU stay $(r=0.47, \mathrm{P}<0.001)$, and hospital stay $(r=0.58, \mathrm{P}<0.001)$. After controlling for the effects of the number of transfused RBCs, the oldest transfused $\mathrm{RBCs}$ were significantly correlated with the length of hospital stay $(r=0.08, \mathrm{P}=0.008)$ but not with the length of ICU stay (Table 4). There was no significant relationship between the other ages of RBCs and the ICU or hospital length of stay (Table 4).

\section{Postoperative base excess}

We evaluated the relationship between RBCs age and base excess on first postoperative morning. After adjusting for the transfusion amount, the oldest transfused RBCs showed a significant negative correlation with the postoperative base excess $(r=-0.08, P=0.02)$. There were no significant relationships between the other ages of RBCs and the base excess (with mean age of transfused RBCs: $\mathrm{r}=-0.03, P=0.32$; with any RBCs unit $>14$ days: $\mathrm{r}=-0.05, P=0.16)$. 
Table 3 The age or the number of transfused RBCs and the postoperative clinical outcomes

\begin{tabular}{|c|c|c|c|c|c|c|c|}
\hline & & & ivariate ar & & & tivariable & \\
\hline & N (\%) & OR & $95 \% \mathrm{Cl}$ & $P$ value & OR & $95 \% \mathrm{Cl}$ & $P$ value \\
\hline Number of transfused RBCs & & & & & & & \\
\hline In-hospital all-cause mortality & $17(1.6)$ & 1.1 & $1.07-1.14$ & $<0.001$ & 1.1 & $1.06-1.14$ & $<0.001$ \\
\hline In-hospital MACCEs & $74(6.9)$ & 1.06 & $1.04-1.09$ & $<0.001$ & 1.05 & $1.03-1.08$ & $<0.001$ \\
\hline New renal failure & $54(5.0)$ & 1.05 & $1.03-1.07$ & $<0.001$ & 1.03 & $1.00-1.05$ & 0.04 \\
\hline Respiratory complication & $33(3.1)$ & 1.05 & $1.03-1.07$ & $<0.001$ & 1.04 & $1.01-1.06$ & 0.005 \\
\hline Postoperative wound complication & $49(4.6)$ & 1.04 & $1.02-1.06$ & $<0.001$ & 1.03 & $1.01-1.06$ & 0.001 \\
\hline Atrial fibrillation & $290(29.1)$ & 1.04 & $1.02-1.06$ & $<0.001$ & 1.02 & $1.00-1.04$ & 0.038 \\
\hline Arrhythmia other than atrial fibrillation & $112(10.4)$ & 1.05 & $1.03-1.07$ & $<0.001$ & 1.03 & $1.02-1.05$ & $<0.001$ \\
\hline Bleeding-related reoperation & $29(2.7)$ & 1.09 & $1.06-1.12$ & $<0.001$ & 1.08 & $1.05-1.12$ & $<0.001$ \\
\hline Oldest age of transfused RBCs & & & & & & & \\
\hline In-hospital all-cause mortality & & 1.1 & $1.02-1.19$ & 0.015 & 1.01 & $0.91-1.12$ & 0.896 \\
\hline In-hospital MACCEs & & 1.01 & $0.97-1.05$ & 0.63 & 0.97 & 0.93-1.02 & 0.198 \\
\hline New renal failure & & 1.08 & $1.03-1.13$ & 0.001 & 1.03 & 0.98-1.09 & 0.251 \\
\hline Respiratory complication & & 1.07 & $1.01-1.13$ & 0.02 & 1.01 & $0.95-1.08$ & 0.726 \\
\hline Postoperative wound complication & & 1.1 & $1.05-1.15$ & $<0.001$ & 1.09 & $1.04-1.14$ & 0.001 \\
\hline Atrial fibrillation & & 1.03 & $1.00-1.05$ & 0.022 & 1.01 & $0.99-1.04$ & 0.339 \\
\hline Arrhythmia other than atrial fibrillation & & 1.04 & $1.00-1.07$ & 0.037 & 1.02 & $0.98-1.05$ & 0.348 \\
\hline Bleeding-related reoperation & & 1.08 & $1.02-1.15$ & 0.007 & 1.02 & $0.95-1.10$ & 0.531 \\
\hline Mean age of transfused RBCs & & & & & & & \\
\hline In-hospital all-cause mortality & & 0.93 & $0.84-1.04$ & 0.23 & 0.95 & 0.82-1.09 & 0.446 \\
\hline In-hospital MACCEs & & 0.95 & $0.90-1.00$ & 0.053 & 0.95 & $0.90-1.01$ & 0.1 \\
\hline New renal failure & & 0.99 & 0.93-1.05 & 0.684 & 1.01 & $0.94-1.08$ & 0.857 \\
\hline Respiratory complication & & 0.99 & $0.92-1.06$ & 0.731 & 0.98 & 0.90-1.06 & 0.629 \\
\hline Postoperative wound complication & & 1.04 & $0.98-1.10$ & 0.201 & 1.05 & $0.99-1.12$ & 0.106 \\
\hline Atrial fibrillation & & 1 & $0.97-1.03$ & 0.969 & 1 & $0.97-1.04$ & 0.783 \\
\hline Arrhythmia other than atrial fibrillation & & 0.99 & $0.95-1.03$ & 0.614 & 1 & $0.96-1.05$ & 0.869 \\
\hline Bleeding-related reoperation & & 1.04 & $0.96-1.12$ & 0.332 & 1.08 & $0.99-1.18$ & 0.094 \\
\hline Any $R B C s$ unit $>14$ days & & & & & & & \\
\hline In-hospital all-cause mortality & & 1.83 & $0.64-4.84$ & 0.225 & 0.7 & $0.20-2.45$ & 0.278 \\
\hline In-hospital MACCEs & & 1.02 & $0.64-1.64$ & 0.933 & 0.74 & $0.44-1.24$ & 0.256 \\
\hline New renal failure & & 1.5 & $0.87-2.60$ & 0.148 & 1.05 & $0.57-1.97$ & 0.869 \\
\hline Respiratory complication & & 1.54 & 0.77-3.09 & 0.224 & 1.06 & $0.50-2.27$ & 0.878 \\
\hline Postoperative wound complication & & 1.96 & $1.10-3.50$ & 0.023 & 1.71 & 0.93-3.12 & 0.083 \\
\hline Atrial fibrillation & & 1.2 & $0.92-1.58$ & 0.18 & 1.06 & $0.80-1.41$ & 0.675 \\
\hline Arrhythmia other than atrial fibrillation & & 1.41 & $0.95-2.09$ & 0.085 & 1.19 & $0.79-1.80$ & 0.407 \\
\hline Bleeding-related reoperation & & 2.24 & $1.05-4.75$ & 0.036 & 1.21 & $0.50-2.93$ & 0.669 \\
\hline
\end{tabular}

RBC, red blood cell; MACCE, major adverse cardiovascular and cerebral event.

Table 4 Correlation between the transfused RBCs and intubated time, ICU stay and Hospital stay

\begin{tabular}{|c|c|c|c|c|c|c|c|c|}
\hline & \multicolumn{2}{|c|}{ Transfusion amout } & \multicolumn{2}{|c|}{ Oldest RBCs age } & \multicolumn{2}{|c|}{ Mean RBCs age } & \multicolumn{2}{|c|}{ Any RBCs unit $>14$ days } \\
\hline & $r$ & $P$ value & $r$ & $P$ value & $r$ & $P$ value & $r$ & $P$ value \\
\hline ICU stay & 0.47 & $<0.001$ & -0.001 & 0.971 & -0.005 & 0.862 & 0.001 & 0.976 \\
\hline Hospital stay & 0.58 & $<0001$ & 0.081 & 0.008 & 0.007 & 0.811 & 0.059 & 0.056 \\
\hline
\end{tabular}

RBC, red blood cell; ICU, intensive care unit. 


\section{Postoperative total bilirubin}

We evaluated the relationship between RBCs age and the postoperative highest total bilirubin as an indicator of hemolysis. Postoperative highest total bilirubin was significantly correlated with the total number of transfused RBCs ( $\mathrm{r}=0.19, \mathrm{P}<0.001)$ and the oldest age of transfused RBCs ( $\mathrm{r}=0.12, \mathrm{P}<0.001)$. However, after adjusting for the transfusion amount, the oldest age of transfused RBCs was not significantly related with the postoperative highest total bilirubin $(r=0.06, \mathrm{P}=0.056)$.

\section{Oldest RBCs age and wound complications}

As there was a significant association between the oldest transfused RBCs and postoperative wound complications, we constructed an additional adjusted model for postoperative wound complications including the oldest age of transfused RBCs as quartiles rather than a continuous variable. In the adjusted model, a significant linear trend was observed between the oldest age quartiles of transfused RBCs and postoperative wound complications (quartile 1 vs. 2: OR, 8.92; 95\% CI, 1.15-69.05; quartile 1 vs. 3: OR, 12.01; 95\% CI, 1.56-92.63; quartile 1 vs. 4: OR, 13.79; 95\% CI, 1.82-104.76; P for trend $=0.009$ ) (Table 5).

There were two patients with other postoperative infections other than wound and the details of those patients were presented in the Additional file 3. We also calculated the postoperative Sepsis related Organ Failure (SOFA) scores [13] and analyzed the relationship with RBCs ages. The total number of transfused RBCs and the oldest age of the transfused RBCs showed significant correlations with the postoperative highest SOFA scores (see Additional file 4).

\section{Discussion}

In this retrospective study, postoperative in-hospital outcome and long-term MACCEs were associated with the amount of transfused RBC units in patients undergoing $\mathrm{OPCAB}$. After adjusting for confounding factors, the age of transfused $\mathrm{RBC}$ units was not associated with longterm MACCEs, but was associated with postoperative wound complications, postoperative day 1 negative base excess, and the length of hospital stay. A linear trend was observed between the oldest age quartiles of transfused RBCs and postoperative wound complications.

Several studies have investigated the clinical effects of RBC storage time in patients undergoing cardiac surgery, including coronary artery bypass grafting (CABG). In a previous retrospective analysis of 3,597 CABG patients, the storage time of transfused RBCs was not a significant predictor of early or late mortality [7] and in other retrospective analyses in CABG patients $[5,8,14,15]$, early postoperative morbidities or major adverse cardiovascular events were not associated with RBCs age using various methods of analysis. The results of the present study are consistent with those earlier results in that the significant adverse effects of older RBCs observed in an unadjusted model disappeared after adjusting for confounding factors. However, our study evaluated the adverse effects of older blood on postoperative wound complications while the previous studies did not.

In contrast to our results, a retrospective study that included 6,002 cardiac surgery patients found that transfusion of RBCs older than 14 days increased in-hospital mortality and mechanical ventilation time, caused various complications, and decreased long-term survival after cardiac surgery [9]. However, several baseline characteristics that were significantly different between the newer and older blood groups (e.g., more left ventricular dysfunction, NYHA class IV, mitral regurgitation, and peripheral vascular disease in the older blood group) may have affected the results. There was no difference in the incidence of sternal wound complications between the two groups in that study; however, approximately half of the patients received transfusions of leukoreduced RBCs and their study population included an unknown ratio of on- and off-pump surgery patients. These

Table 5 Predictors for postoperative wound complication

\begin{tabular}{|c|c|c|c|c|}
\hline & \multicolumn{4}{|c|}{ Adjusted model } \\
\hline & OR & $95 \% \mathrm{Cl}$ & $P$ value & $P$ for trend ${ }^{*}$ \\
\hline DM & 1.94 & $1.04-3.63$ & 0.038 & \\
\hline BMl & 1.13 & $1.03-1.25$ & 0.011 & \\
\hline Number of transfused RBCs & 1.04 & $1.02-1.06$ & $<0.001$ & \\
\hline Oldest age quartile 1 of transfused $\mathrm{RBCs}^{*}$ (reference) & & & 0.067 & 0.009 \\
\hline quartile 2 & 8.92 & $1.15-69.05$ & 0.036 & \\
\hline quartile 3 & 12.01 & $1.56-92.63$ & 0.017 & \\
\hline quartile 4 & 13.79 & $1.82-104.76$ & 0.011 & \\
\hline
\end{tabular}

${ }^{*} \mathrm{P}$ for trend refers to linear trend across lowest to highest quartile.

*Oldest age quartile 1: oldest age of transfused RBCs unit $<10$ days ( $n=218$ ), quartile 2: oldest age of transfused RBCs unit $<14$ days ( $n=304$ ), quartile 3 : oldest age of transfused RBCs unit $<18$ days $(n=254)$, quartile 4: oldest age of transfused RBCs unit $\geq 18$ days $(n=296)$.

RBC, red blood cell; DM, diabetes mellitus; BMI, body mass index. 
differences complicated comparing their results with our results. Another study reported a significant association between RBC storage age and the morbidity and mortality after cardiac surgery [16], but it was voluntarily retracted.

Based on the results of many previous studies of the effects of RBCs storage, a storage time of more than 14 days is considered hazardous; thus, 14 days is often used as the cutoff point for patient grouping [7,9,15,17]. The levels of intracellular 2,3-DPG decline to undetectable levels by storage day $14[3,18]$ but begin to normalize within a few hours of transfusion and are completely restored within $72 \mathrm{~h}$ after transfusion $[19,20]$.

The adverse effects of RBC transfusion on postoperative morbidity and mortality have been demonstrated in numerous studies [6,21-23]. In this study, the number of perioperative RBCs transfusions was associated with in-hospital and long-term clinical outcomes, consistent with previous studies. On the other hand, the clinical adverse effects of storage time of RBCs disappeared after adjusting for confounding factors including transfusion amount, possibly due to RBCs breakdown that occurs during RBCs processing regardless of RBCs age [24].

A dose-dependent adverse effect of RBCs transfusions on postoperative wound complications has been reported in numerous studies and increased body mass index and diabetes mellitus have been identified as additional risk factors [25-28]; our results are consistent with these findings. Moreover, we found that the oldest age of transfused RBCs was also associated with increased postoperative wound complications. Although it is difficult to determine the causal relationship, significant relationships were observed between the oldest transfused RBCs and the postoperative negative base excess $(r=-0.079$, $\mathrm{P}=0.02$ ), between negative base excess and postoperative wound complications $(\mathrm{r}=-0.086, \mathrm{P}=0.01)$, and between postoperative wound complications and prolonged hospital stay $(\mathrm{r}=0.28, \mathrm{P}<0.001)$. Monitoring of the intra- and postoperative acid-base status has been used as a surrogate marker of tissue oxygen delivery and cellular perfusion [29]. The negative base excess was significantly correlated with the oldest age of transfused RBCs units, which may be due to insufficient oxygen supply to the tissues. This may suggest that older blood transfusions induce tissue hypoperfusion, which increases postoperative wound complications, consequently leading to a prolonged hospital stay.

Moreover, reduced bioavailability of nitric oxide in older RBCs units might have delayed the wound healing [3]. A number of studies investigated the role of nitric oxide in wound healing [30-33]. Synthesis of nitric oxide occurs during wound healing, especially in the early stage of healing [30], and the released nitric oxide improved wound repair by angiogenesis, collagen formation, cell proliferation, and fibroblast migration in damaged tissue
[31-33]. Possible other explanation for increased wound complications was transfusion-related immunomodulation in the blood recipients, especially those receiving nonleukoreduced RBCs [34]. A previous prospective cohort study with trauma patients reported that prestorage leukoreduction abrogated the detrimental effect of old stored blood [35]. Although the different characteristics of the study population make it difficult to compare the findings of that study with our results, leukoreduction might have reduced the wound infection in our study.

This study had several limitations. Our study population contained only coronary disease patients who underwent OPCAB by a single surgeon in a single tertiary center. Therefore, it was relatively more homogeneous than populations in previous studies of cardiac surgery patients. However, the retrospective design means that uncontrolled biases could have affected our analyses. As it is difficult to randomize patients to receive older blood transfusions due to ethical concerns and technical problems, the results of some randomized controlled trials of cardiac surgery patients (ClinicalTrials.gov No.: NCT00458783 and NCT00991341) [36] awaiting publication will be important in drawing conclusions about the effects of old stored RBCs on various clinical outcomes.

For another limitation, all transfused RBCs in this study were from nonleukocyte-depleted blood and the clinical effects of stored leukocyte-depleted or irradiated RBCs remain unclear. Filtered RBCs unit showed different level of RBCs hemolysis and leukocyte breakdown from unfiltered RBCs unit over time [24], and gamma irradiation increases hemolysis of RBCs causing potassium leakage and liberates reactive oxygen species. Thus, further studies are needed to identify the clinical effects of those RBCs unit.

\section{Conclusions}

Postoperative in-hospital outcome and long-term MACCEs were associated with the amount of transfused RBC units. The oldest transfused RBC units were associated with the greatest incidence of postoperative wound complications, negative base excess, and longest duration of hospital stay, but not with the other in-hospital outcomes or long-term MACCEs. This suggests that hypoperfused tissue caused by the transfusion of older stored blood increases the incidence of postoperative wound complications, consequently increasing the hospital stay.

\section{Additional files}

Additional file 1: Detailed description of long-term MACCEs.

Additional file 2: Predictors for postoperative clinical outcome.

Additional file 3: Postoperative infections other than wound.

Additional file 4: Postoperative SOFA score and RBCs age. 


\section{Abbreviations}

RBC: Red blood cell; OPCAB: Off-pump coronary artery bypass; MACCE: Major adverse cardiovascular and cerebral event; ICU: Intensive care unit; CABG: Coronary artery bypass grafting.

\section{Competing interests}

The authors declare that they have no competing interests.

\section{Authors' contributions}

YJ, JJM, KSH, DMH designed and coordinated the study. YJ, JJM, JYB, $\mathrm{HYH}, \mathrm{KBK}, \mathrm{KSH}$ collected patient data. YJ, DMH, JJM, JYB, TKK analyzed the data. JJM, JYB, TKK prepared manuscript and submitted article. YJ, HYH, $\mathrm{KBK}, \mathrm{DMH}, \mathrm{KSH}$ contributed to data interpretation and revision of the manuscript. YJ was the principal investigator and the cardiothoracic anesthesiologist. All authors have seen and approved the final version of the manuscript.

\section{Acknowledgements}

The authors thank to the Medical Research Collaborating Center (MRCC) of Seoul National University Hospital for the statistical assistance and supervision.

\section{Author details}

1Department of Anesthesiology and Pain Medicine, Seoul National University Hospital, Seoul, Korea. '2Department of Anesthesiology and Pain Medicine, Samsung Medical Center, Sungkyunkwan University School of Medicine, Seoul, Korea. ${ }^{3}$ Department of Thoracic and Cardiovascular Surgery, Seoul National University Hospital, Seoul, Korea. ${ }^{4}$ Department of Laboratory Medicine, Seoul National University Hospital, Seoul, Korea.

Received: 4 June 2014 Accepted: 14 October 2014 Published: 21 October 2014

\section{References}

1. Kim-Shapiro DB, Lee J, Gladwin MT: Storage lesion: role of red blood cell breakdown. Transfusion 2011, 51(4):844-851.

2. Doctor A, Spinella P: Effect of processing and storage on red blood cell function in vivo. Semin Perinatol 2012, 36(4):248-259.

3. Roback JD: Vascular effects of the red blood cell storage lesion. Hematology Am Soc Hematol Educ Program 2011, 2011:475-479.

4. Van de Watering L: Red cell storage and prognosis. Vox Sang 2011, 100(1):36-45.

5. Van de Watering L, Lorinser J, Versteegh $M$, Westendord R, Brand A: Effects of storage time of red blood cell transfusions on the prognosis of coronary artery bypass graft patients. Transfusion 2006, 46(10):1712-1718.

6. McKenny M, Ryan T, Tate H, Graham B, Young VK, Dowd N: Age of transfused blood is not associated with increased postoperative adverse outcome after cardiac surgery. Br J Anaesth 2011, 106(5):643-649.

7. Van Straten AH, Soliman Hamad MA, Van Zundert AA, Martens EJ, Ter Woorst JF, de Wolf AM, Scharnhorst V: Effect of duration of red blood cell storage on early and late mortality after coronary artery bypass grafting. J Thorac Cardiovasc Surg 2011, 141(1):231-237.

8. Yap CH, Lau L, Krishnaswamy M, Gaskell M, Yii M: Age of transfused red cells and early outcomes after cardiac surgery. Ann Thorac Surg 2008, 86(2):554-559.

9. Koch CG, Li L, Sessler DI, Figueroa P, Hoeltge GA, Mihaljevic T, Blackstone $\mathrm{EH}$ : Duration of red-cell storage and complications after cardiac surgery. N Engl J Med 2008, 358(12):1229-1239.

10. Esper SA, Subramaniam K, Tanaka KA: Pathophysiology of cardiopulmonary bypass: current strategies for the prevention and treatment of anemia, coagulopathy, and organ dysfunction. Semin Cardiothorac Vasc Anesth 2014, 18(2):161-176.

11. Sweeney J, Kouttab N, Kurtis J: Stored red blood cell supernatant facilitates thrombin generation. Transfusion 2009, 49(8):1569-1579.

12. Lamy A, Devereaux PJ, Prabhakaran D, Taggart DP, Hu S, Paolasso E, Straka Z, Piegas LS, Akar AR, Jain AR, Noiseux N, Padmanabhan C, Bahamondes JC, Novick RJ, Vaijyanath P, Reddy S, Tao L, Olavegogeascoechea PA, Airan B, Sulling TA, Whitlock RP, Ou Y, Ng J, Chrolavicius S, Yusuf S: Off-pump or on-pump coronary-artery bypass grafting at 30 days. N Engl J Med 2012, 366(16):1489-1497.
13. Vincent JL, Moreno R, Takala J, Willatts S, De Mendonca A, Bruining H, Reinhart CK, Suter PM, Thijs LG: The SOFA (Sepsis-related Organ Failure Assessment) score to describe organ dysfunction/failure: on behalf of the working group on sepsis-related problems of the European Society of Intensive Care Medicine. Intensive Care Med 1996, 22(7):707-710.

14. Vamvakas EC, Carven JH: Length of storage of transfused red cells and postoperative morbidity in patients undergoing coronary artery bypass graft surgery. Transfusion 2000, 40(1):101-109.

15. Voorhuis FT, Dieleman JM, de Vooght KM, van Dijk D, van Herwerden LA, Peelen LM, van Klei WA: Storage time of red blood cell concentrates and adverse outcomes after cardiac surgery: a cohort study. Ann Hematol 2013, 92(12):1701-1706.

16. Basran S, Frumento RJ, Cohen A, Lee S, Du Y, Nishanian E, Kaplan HS, Stafford-Smith M, Bennett-Guerrero E: The association between duration of storage of transfused red blood cells and morbidity and mortality after reoperative cardiac surgery. Anesth Analg 2006, 103(1):15-20. table of contents.

17. Weinberg JA, McGwin G Jr, Griffin RL, Huynh VQ, Cherry SA III, Marques MB, Reiff DA, Kerby JD, Rue LW III: Age of transfused blood: an independent predictor of mortality despite universal leukoreduction. J Trauma 2008, 65(2):279-282. discussion 282-274.

18. Almac $E$, Ince $C$ : The impact of storage on red cell function in blood transfusion. Best Pract Res Clin Anaesthesiol 2007, 21(2):195-208.

19. Steiner ME, Stowell C: Does red blood cell storage affect clinical outcome? When in doubt, do the experiment. Transfusion 2009 49(7):1286-1290.

20. Kor DJ, Van Buskirk CM, Gajic O: Red blood cell storage lesion. Bosn J Basic Med Sci 2009, 9 Suppl 1:21-27.

21. Koch CG, Li L, Duncan Al, Mihaljevic T, Loop FD, Starr NJ, Blackstone EH: Transfusion in coronary artery bypass grafting is associated with reduced long-term survival. Ann Thorac Surg 2006, 81(5):1650-1657.

22. Koch CG, Li L, Van Wagoner DR, Duncan Al, Gillinov AM, Blackstone EH: Red cell transfusion is associated with an increased risk for postoperative atrial fibrillation. Ann Thorac Surg 2006, 82(5):1747-1756.

23. Koch CG, Li L, Duncan Al, Mihaljevic T, Cosgrove DM, Loop FD, Starr NJ, Blackstone EH: Morbidity and mortality risk associated with red blood cell and blood-component transfusion in isolated coronary artery bypass grafting. Crit Care Med 2006, 34(6):1608-1616.

24. Sowemimo-Coker SO: Red blood cell hemolysis during processing Transfus Med Rev 2002, 16(1):46-60

25. Vymazal T, Horacek M, Durpekt R, Hladikova M, Cvachovec K: Is allogeneic blood transfusion a risk factor for sternal dehiscence following cardiac surgery? A prospective observational study. Int Heart J 2009, 50(5):601-607

26. Banbury MK, Brizzio ME, Rajeswaran J, Lytle BW, Blackstone EH: Transfusion increases the risk of postoperative infection after cardiovascular surgery. J Am Coll Surg 2006, 202(1):131-138.

27. Friedman ND, Bull AL, Russo PL, Leder K, Reid C, Billah B, Marasco S, McBryde E, Richards MJ: An alternative scoring system to predict risk for surgical site infection complicating coronary artery bypass graft surgery. Infect Control Hosp Epidemiol 2007, 28(10):1162-1168.

28. Andreasen JJ, Dethlefsen C, Modrau IS, Baech J, Schonheyder HC, Moeller JK, Johnsen SP: Storage time of allogeneic red blood cells is associated with risk of severe postoperative infection after coronary artery bypass grafting. Eur J Cardiothorac Surg 2011, 39(3):329-334.

29. Allen M: Lactate and acid base as a hemodynamic monitor and markers of cellular perfusion. Pediatr Crit Care Med 2011, 12(4 Suppl):S43-S49.

30. Witte MB, Barbul A: Role of nitric oxide in wound repair. Am J Surg 2002, 183(4):406-412.

31. Schwentker A, Vodovotz $Y$, Weller R, Billiar TR: Nitric oxide and wound repair: role of cytokines? Nitric Oxide 2002, 7(1):1-10.

32. Luo JD, Chen AF: Nitric oxide: a newly discovered function on wound healing. Acta Pharmaco/ Sin 2005, 26(3):259-264

33. Han G, Nguyen LN, Macherla C, Chi Y, Friedman JM, Nosanchuk JD, Martinez LR: Nitric oxide-releasing nanoparticles accelerate wound healing by promoting fibroblast migration and collagen deposition. Am J Pathol 2012, 180(4):1465-1473.

34. Vamvakas EC, Blajchman MA: Transfusion-related immunomodulation (TRIM): an update. Blood Rev 2007, 21(6):327-348.

35. Phelan HA, Eastman AL, Aldy K, Carroll EA, Nakonezny PA, Jan T, Howard JL, Chen Y, Friese RS, Minei JP: Prestorage leukoreduction abrogates the 
detrimental effect of aging on packed red cells transfused after trauma: a prospective cohort study. Am J Surg 2012, 203(2):198-204.

36. Steiner ME, Assmann SF, Levy JH, Marshall J, Pulkrabek S, Sloan SR, Triulzi D, Stowell CP: Addressing the question of the effect of RBC storage on clinical outcomes: the Red Cell Storage Duration Study (RECESS) (Section 7). Transfus Apher Sci 2010, 43(1):107-116.

doi:10.1186/1471-2253-14-95

Cite this article as: Min et al: Association between red blood cell storage duration and clinical outcome in patients undergoing off-pump coronary artery bypass surgery: a retrospective study. BMC Anesthesiology 2014 14:95.

\section{Submit your next manuscript to BioMed Central and take full advantage of:}

- Convenient online submission

- Thorough peer review

- No space constraints or color figure charges

- Immediate publication on acceptance

- Inclusion in PubMed, CAS, Scopus and Google Scholar

- Research which is freely available for redistribution 